\title{
Variable Stability Heterodimeric Coiled-Coils from Manipulation of Electrostatic Interface Residue Chain Length
}

\author{
Shannon J. Ryan and Alan J. Kennan* \\ Department of Chemistry, Colorado State University Fort Collins, Colorado 80523
}

\section{Supporting Information:}

\section{General Procedures:}

Peptide Synthesis. Amino acids (except for HGlu, HHGlu) were obtained from NovaBiochem (San Diego) or Bachem (Torrance, CA). HGlu and HHGlu were synthesized as described previously. ${ }^{1}$ Peptides were prepared according to the in situ neutralization protocol ${ }^{2}$ developed by Kent, except for pHGlu and pHHGlu which were prepared by standard Fmoc methods. Each peptide was purified by reverse-phase HPLC (C-18 column, solvent A: $1 \% \mathrm{CH}_{3} \mathrm{CN}$ in $\mathrm{H}_{2} \mathrm{O}$, $0.1 \%(\mathrm{v} / \mathrm{v}) \mathrm{CF}_{3} \mathrm{CO}_{2} \mathrm{H}$; solvent $\mathrm{B}: 10 \% \mathrm{H}_{2} \mathrm{O}$ in $\mathrm{CH}_{3} \mathrm{CN}, 0.07 \%(\mathrm{v} / \mathrm{v}) \mathrm{CF}_{3} \mathrm{CO}_{2} \mathrm{H}$ ), and the identity of purified samples was confirmed by electrospray mass spectrometry (Finnegan LCQ-Duo). All peptides are C-terminally amidated and N-terminally acetylated; each contains an acetamidobenzoate group on the sidechain nitrogen of a solvent-exposed lysine as a spectroscopic label.

Ni-NTA Affinity Tag Experiments. A $0.5 \mathrm{~mL}$ sample of a 50\% slurry of Ni-NTA agarose (Qiagen) in an Eppendorf tube was centrifuged for $30 \mathrm{~s}$, followed by removal of the superantant. Peptide solution (10 $\mu \mathrm{M}$ total peptide) was added, and the tube was repeatedly inverted for 5 minutes. The sample was centrifuged (30 s) and the supernatant (flow-through fraction) was removed. The procedure was then repeated with $1 \mathrm{~mL}$ of buffer (wash fraction) and $1 \mathrm{~mL}$ of buffer containing $250 \mathrm{mM}$ imidazole (elution fraction), except that the wash fraction was only agitated for 30 seconds. $^{3}$ Exchange experiments involved two consecutive flow-through/wash cycles before elution, the first solution containing the original complex and the second containing one equivalent of the new peptide. Solutions were analyzed by RP-HPLC.

CD Spectroscopy. All experiments were performed on an Aviv model 202 circular dichroism spectrometer, equipped with a Microlab 500 series automated titration assembly. Sample concentrations were measured by UV absorbance of the acetamidobenzoate label at $270 \mathrm{~nm}$. Wavelength data are the average of three scans from 250 to $200 \mathrm{~nm}$ in $1 \mathrm{~nm}$ steps. Thermal denaturation experiments at $222 \mathrm{~nm}$ were run from $0^{\circ}$ to $90^{\circ} \mathrm{C}$ in two-degree steps, at a twodegrees/minute rate of increase with one-minute equilibration and data averaging at each temperature. $T_{m}$ values were obtained from minima of the first derivative of $\theta \mathrm{vs.} 1 / \mathrm{T}_{\text {plots. }}{ }^{4}$

(1) Ryan, S. J.; Zhang, Y.; Kennan, A. J. Org. Lett., 2005, 7, 4765-4767.

(2) Schnöelzer, M.; Alewood, P.; Jones, A.; Alewood, D.; Kent, S. B. H. Int. J. Pept. Protein Res. 1992, 40, 180-193.

(3) Method patterned after that in: Brown, M. B.; Sauer, R. T. Proc. Nat. Acad. Sci. U.S.A., 1999, 96, 1983-1988.

(4) Cantor, C. R.; Schimmel, P. R. Biophysical Chemistry of Macromolecules, Pt. 3: The Behavior of Biological Macromolecules; W. H. Freeman: New York, NY, 1980, p.1132. 
Analytical Ultracentrifugation. Sedimentation equilibrium experiments were performed using a Beckman XL-I or XL-A analytical ultracentrifuge equipped with an An60-Ti or An50-Ti rotor. Data were collected using $12 \mathrm{~mm}$ path length six-sector centerpieces at $270 \mathrm{~nm}$. Samples were dialyzed against the reference buffer at $4^{\circ} \mathrm{C}$ overnight. Data were collected at 38,000 and 48,000 r.p.m. at concentrations spanning 17-55 $\mu \mathrm{M}$. Samples were judged equilibrated when three consecutive scans taken $1 \mathrm{~h}$ apart were indistinguishable (equilibration was complete in $12 \mathrm{~h}$ in all cases). Solvent densities and partial molar volumes were calculated in the manner prescribed by Laue, ${ }^{5}$ except for pHGlu and pHHGlu (see discussion below). Data were analyzed using Ultrascan ${ }^{6}$ and fit globally to appropriate models.

\section{Additional Ni-NTA data:}

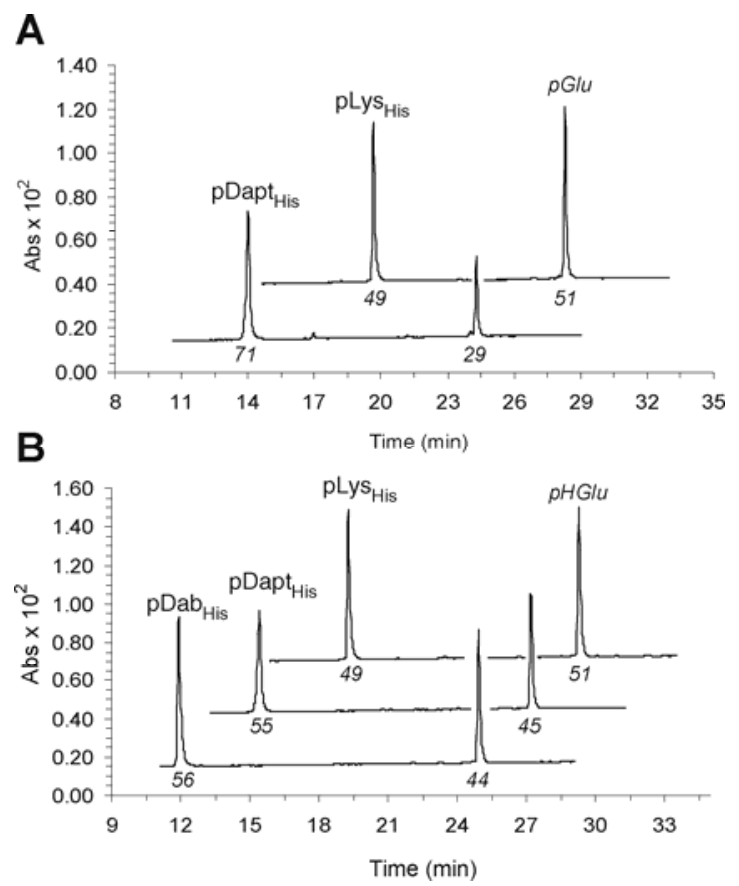

Figure S-1 Ni-NTA analysis of heterodimer complex stoichiometry. HPLC traces of elution fractions from initial 1:1 mixtures of basic peptides with pGlu (A) and pHGlu (B), as in manuscript Figure 5. Ratios of peak areas are given as relative percentages (corresponding ratios for Figure 5 are: 55:45, 53:47, 50:50, for $\mathrm{pDab}_{\mathrm{His}} \mathrm{pDapt}_{\mathrm{His}}$ and $\mathrm{pLys}_{\mathrm{His}}$ with $\mathrm{pHHGlu}$ ).

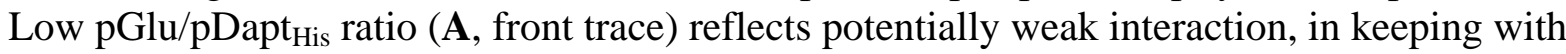
CD data.

(5) Laue, T. M.; Shah, B. D.; Ridgeway, T. M.; Pelletier, S. L. in Analytical Ultracentrifugation in Biochemistry and Polymer Science; Harding, S. E., Rowe, A. J., Horton, J. C., Eds.; The Royal Society of Chemistry: Cambridge, 1992; pp 90-125.

(6) Ultrascan (version 7.1). Developed by Borries Demeler, The University of Texas Health Science Center at San Antonio. 


\section{Additional CD data:}

Traces are given for pure components and calculated weighted averages, compared to those for heterodimer solutions. Significant deviations from weighted average spectra imply increased helical content in equimolar mixtures, presumably due to complex formation.
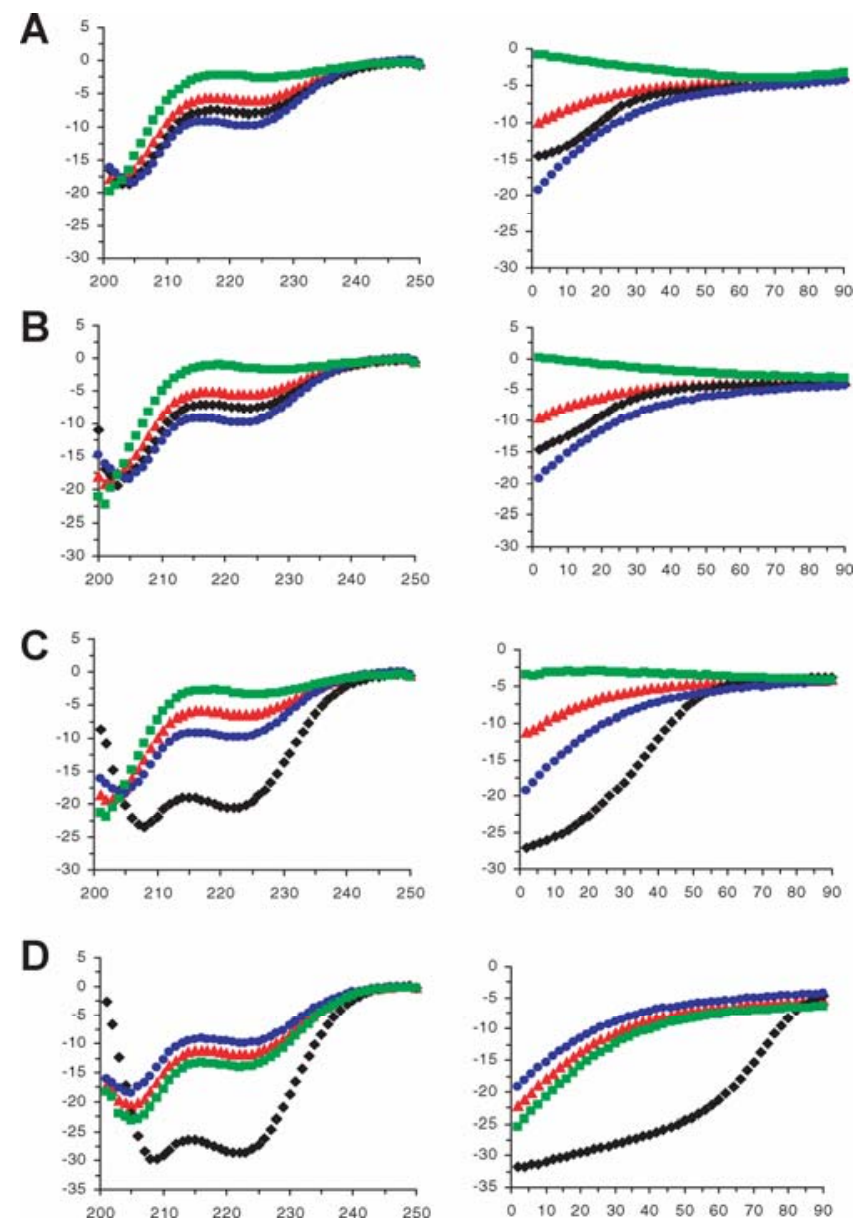

Figure S-2 Wavelength (left) and thermal denaturation (right) CD spectra for interactions of pGlu with pDap (A), pDab (B), pDapt (C) and pLys (D). Each spectrum contains traces for pure pGlu (blue circles), pure basic peptide (green squares), the 1:1 mixture (black diamonds) and the calculated weighted average spectrum (red triangles). Conditions as described above. 

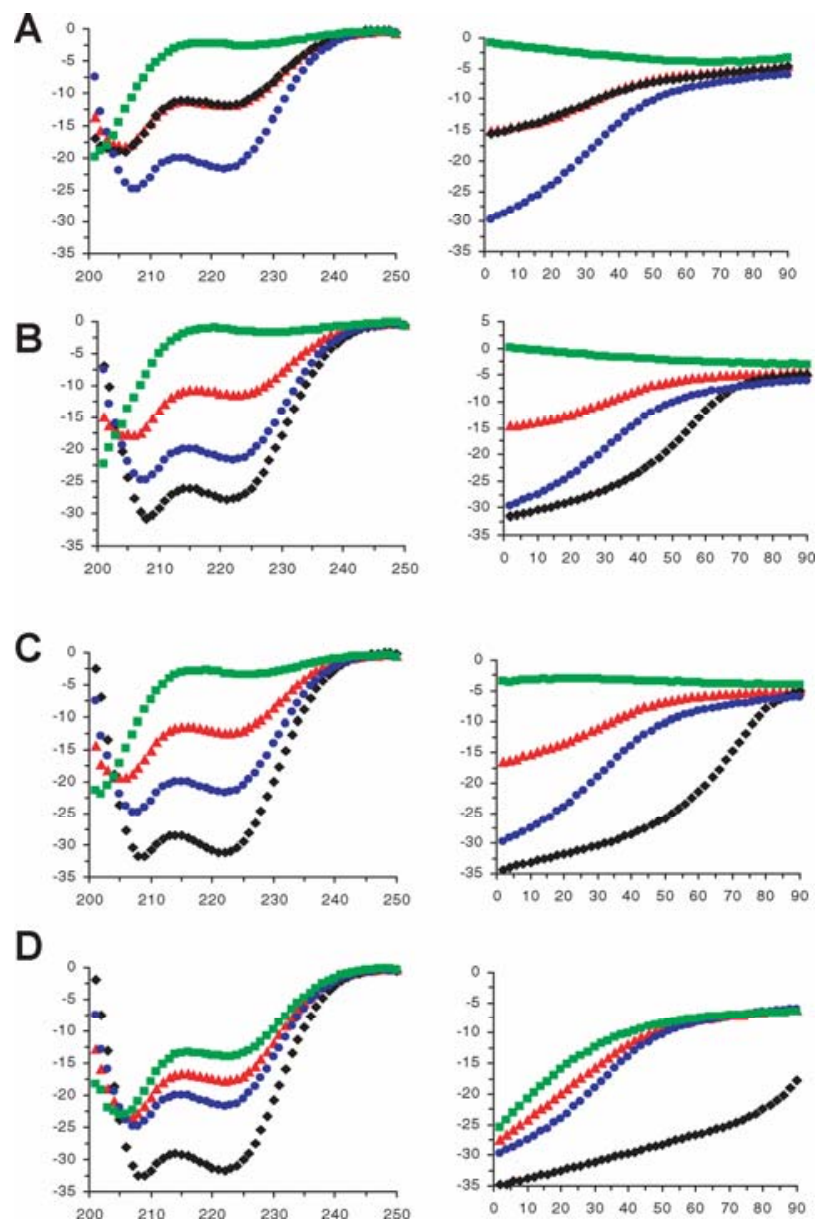

Figure S-3 Wavelength (left) and thermal denaturation (right) CD spectra for interactions of pHGlu with pDap (A), pDab (B), pDapt (C) and pLys (D). Each spectrum contains traces for pure pHGlu (blue circles), pure basic peptide (green squares), the 1:1 mixture (black diamonds) and the calculated weighted average spectrum (red triangles). Conditions as described above. 

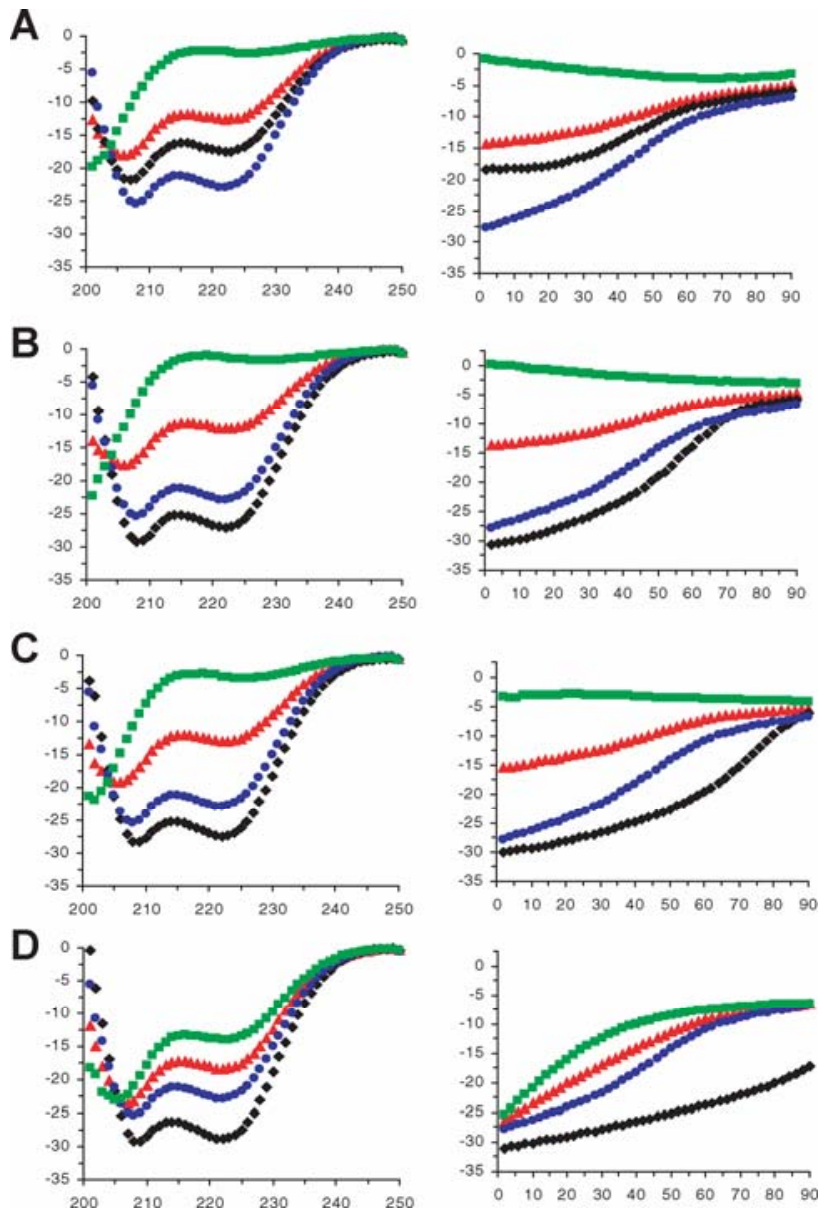

Figure S-4 Wavelength (left) and thermal denaturation (right) CD spectra for interactions of pHHGlu with pDap (A), pDab (B), pDapt (C) and pLys (D). Each spectrum contains traces for pure pHHGlu (blue circles), pure basic peptide (green squares), the 1:1 mixture (black diamonds) and the calculated weighted average spectrum (red triangles). Conditions as described above. 


\section{Additional analytical ultracentrifugation data:}

To estimate partial specific volume (v_bar) values for pDab, pDapt, pHGlu and pHHGlu peptides, fits were carried out using one of three values for each non-proteinogenic monomer: 0.72 (the average v_bar for all natural amino acids), 0.60 (the minimum natural value, for Asp), and 0.90 (the maximum natural value, for Leu/lle). In complexes with two non-proteinogenic monomers one of the two was held at the average value while the other was varied. Below are the results of these fits. The values reported in the manuscript use 0.72 for Dab, Dapt, HGlu and HHGlu.

\begin{tabular}{ccc}
$\begin{array}{c}\text { pDapt/pGlu } \\
\text { Dapt v_bar }\end{array}$ & $\mathrm{M}_{\text {robs }}$ & \% of $\mathbf{M}_{r \text { calc }}$ \\
\hline 0.72 & 6819 & 95 \\
0.60 & 6381 & 89 \\
0.90 & 7602 & 105
\end{tabular}

pHGlu complexes

pHGlu dimer

\begin{tabular}{ccc} 
HGlu v_bar & $\mathrm{M}_{r \text { obs }}$ & \% of $\mathrm{M}_{r \text { calc }}$ \\
\hline 0.72 & 6839 & 91 \\
0.60 & 6009 & 80 \\
0.90 & 8754 & 115
\end{tabular}

\begin{tabular}{ccc} 
pHGlu/pDab & \multicolumn{2}{c}{ HGlu v_bar $=0.72$} \\
Dab v_bar & M $_{\text {robs }}$ & \% of $\mathrm{M}_{r \text { calc }}$ \\
\hline 0.72 & 7603 & 105 \\
0.60 & 7098 & 98 \\
0.90 & 8511 & 118
\end{tabular}

\begin{tabular}{ccc}
$\begin{array}{c}\text { pHGlu/pDapt } \\
\text { Dapt v_bar }\end{array}$ & \multicolumn{2}{c}{ HGlu v_bar $=0.72$} \\
M $_{\text {obs }}$ & \% of $\mathrm{M}_{\text {r calc }}$ \\
\hline 0.72 & 7886 & 108 \\
0.60 & 7362 & 100 \\
0.90 & 8828 & 120
\end{tabular}

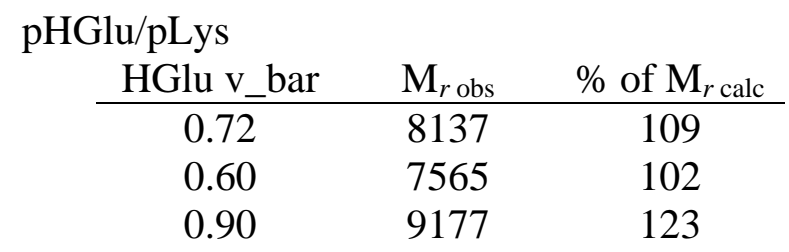




\begin{tabular}{|c|c|c|}
\hline \multicolumn{3}{|c|}{ pHHGlu complexes } \\
\hline pHHGlu dimer & & \\
\hline HHGlu v_bar & $\mathrm{M}_{r \text { obs }}$ & $\%$ of $\mathrm{M}_{r \text { calc }}$ \\
\hline 0.72 & 7152 & 92 \\
\hline 0.60 & 6260 & 80 \\
\hline 0.90 & 9096 & 117 \\
\hline pHHGlu/pDab & \multicolumn{2}{|c|}{ HHGlu v_bar $=0.72$} \\
\hline Dab v_bar & $\mathrm{M}_{\text {robs }}$ & $\%$ of $\mathrm{M}_{r \text { calc }}$ \\
\hline 0.72 & 7818 & 107 \\
\hline 0.60 & 7299 & 100 \\
\hline 0.90 & 8752 & 119 \\
\hline pHHGlu/pDapt & \multicolumn{2}{|c|}{ HHGlu v_bar $=0.72$} \\
\hline Dapt v_bar & $\mathrm{M}_{\text {robs }}$ & $\%$ of $\mathrm{M}_{r \text { calc }}$ \\
\hline 0.72 & 7914 & 106 \\
\hline 0.60 & 7388 & 99 \\
\hline 0.90 & 8859 & 119 \\
\hline \multicolumn{3}{|l|}{ pHHGlu/pLys } \\
\hline HHGlu v_bar & $\mathrm{M}_{\text {robs }}$ & $\%$ of $\mathrm{M}_{r \text { calc }}$ \\
\hline 0.72 & 7837 & 104 \\
\hline 0.60 & 7287 & 97 \\
\hline 0.90 & 8812 & 117 \\
\hline
\end{tabular}




\section{Analytical ultracentrifugation fits:}

Below are the fits used to obtain the values reported in the paper (see above for a discussion of v_bar issues).

\section{pHGlu}

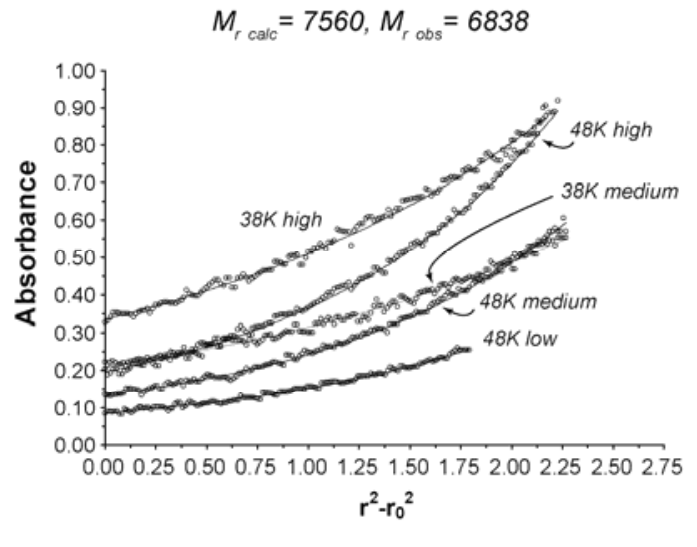

48K low
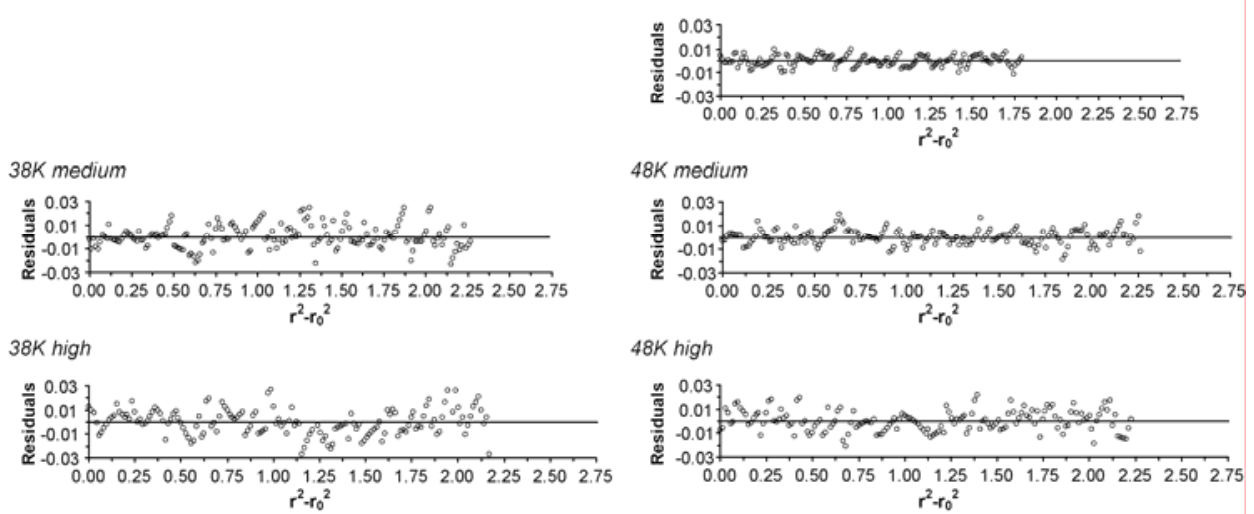


\section{pHHGlu}
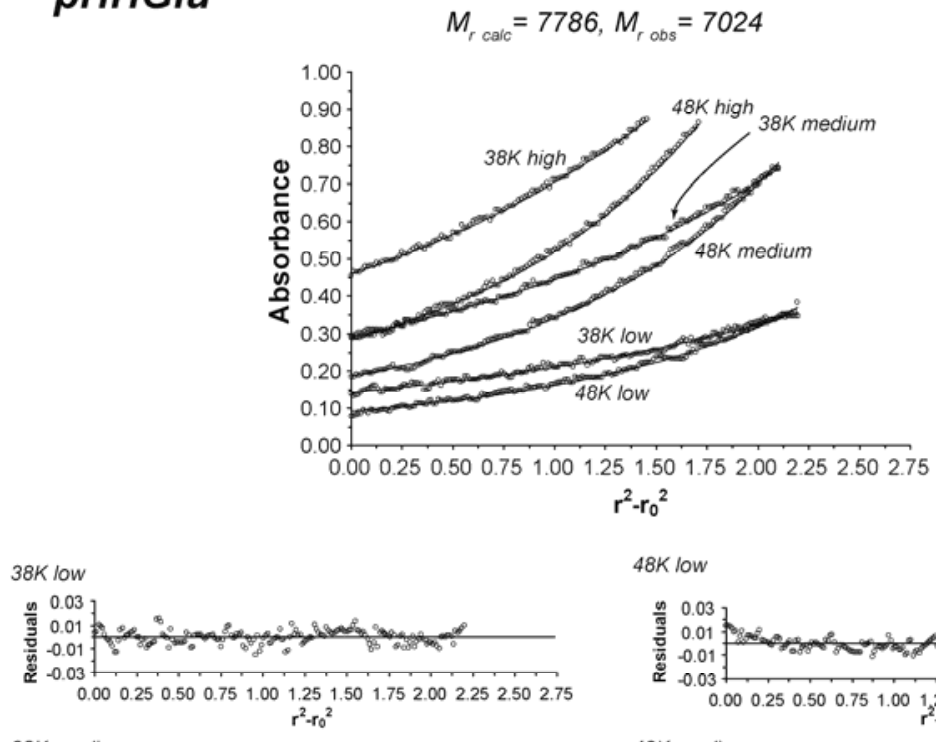

48K low
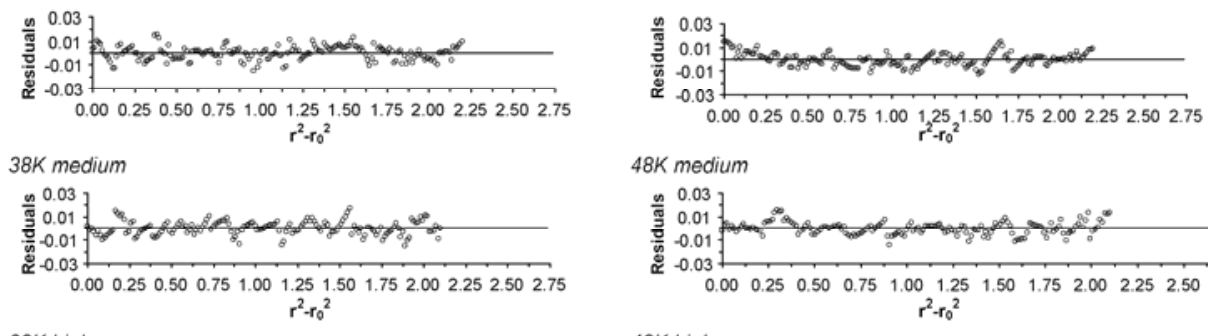

$48 \mathrm{~K}$ medium
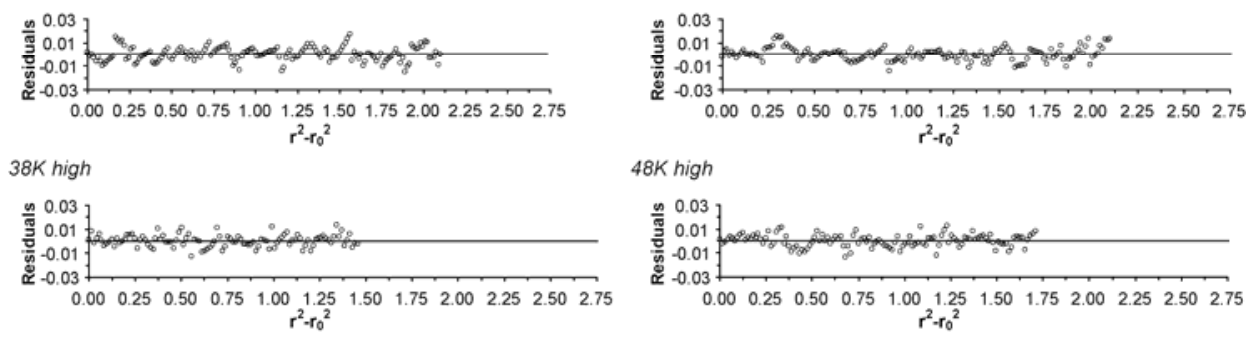

48K high

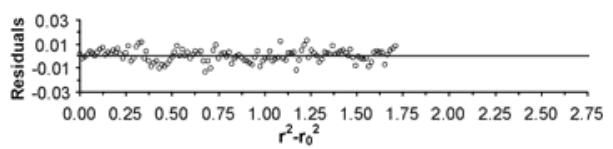


pDab/pHGlu

$M_{r \text { calc }}=7216, M_{r o b s}=7442$

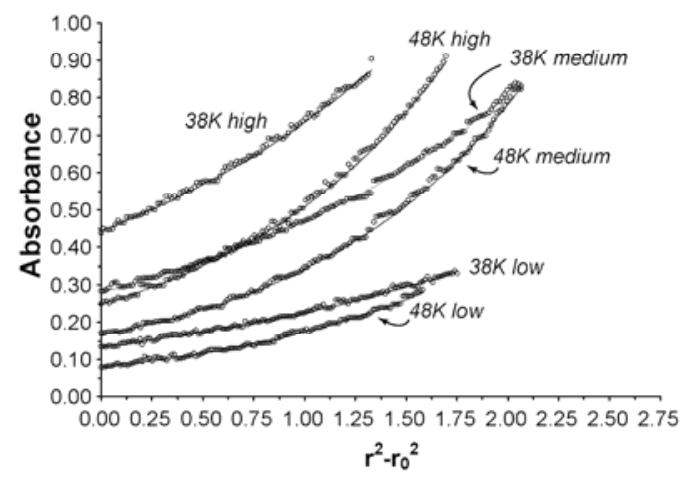

$38 \mathrm{~K}$ low

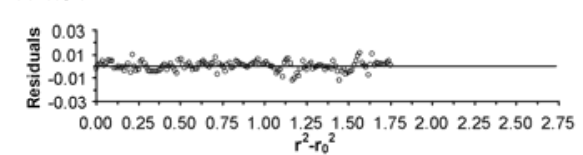

$38 \mathrm{~K}$ medium

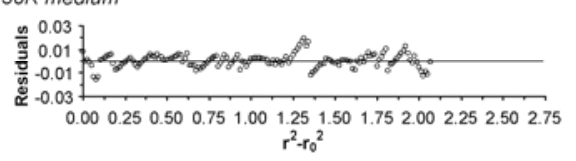

$38 \mathrm{~K}$ high

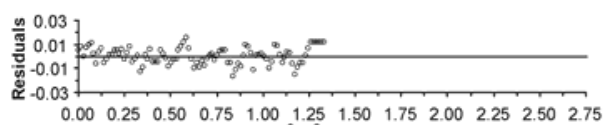

$48 \mathrm{~K} \mathrm{low}$

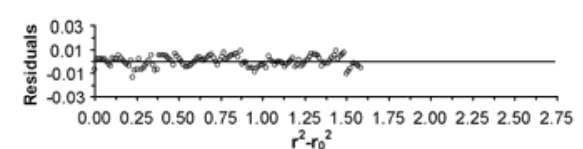

$48 \mathrm{~K}$ medium

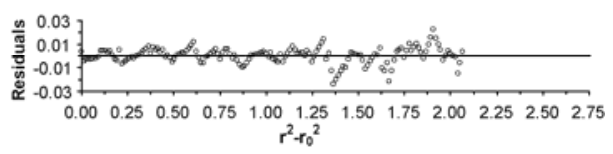

$48 K$ high

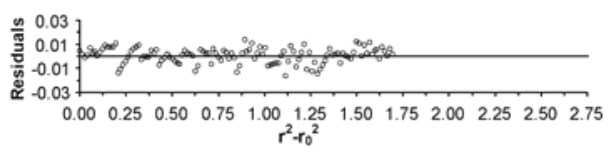




\section{pDapt/pHGlu}
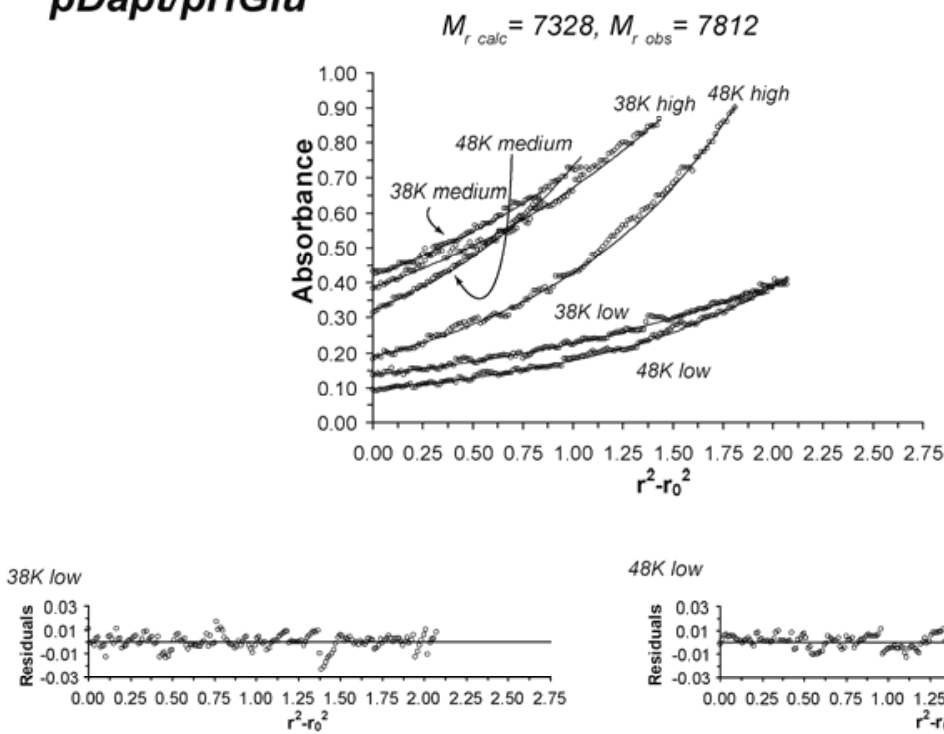

$48 K$ low

$38 \mathrm{~K}$ medium

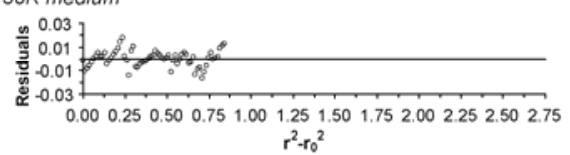

$38 \mathrm{~K}$ high

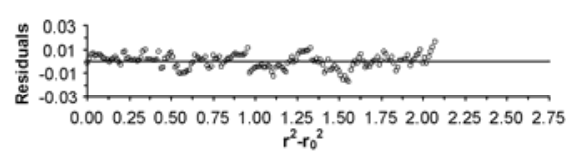

48K medium

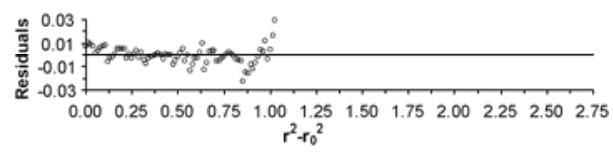

48K high
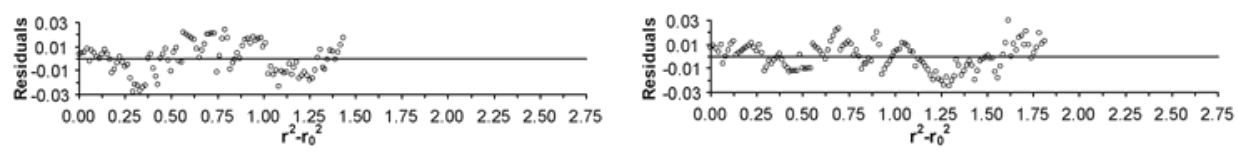

S-11 


\section{pLys/pHGlu}
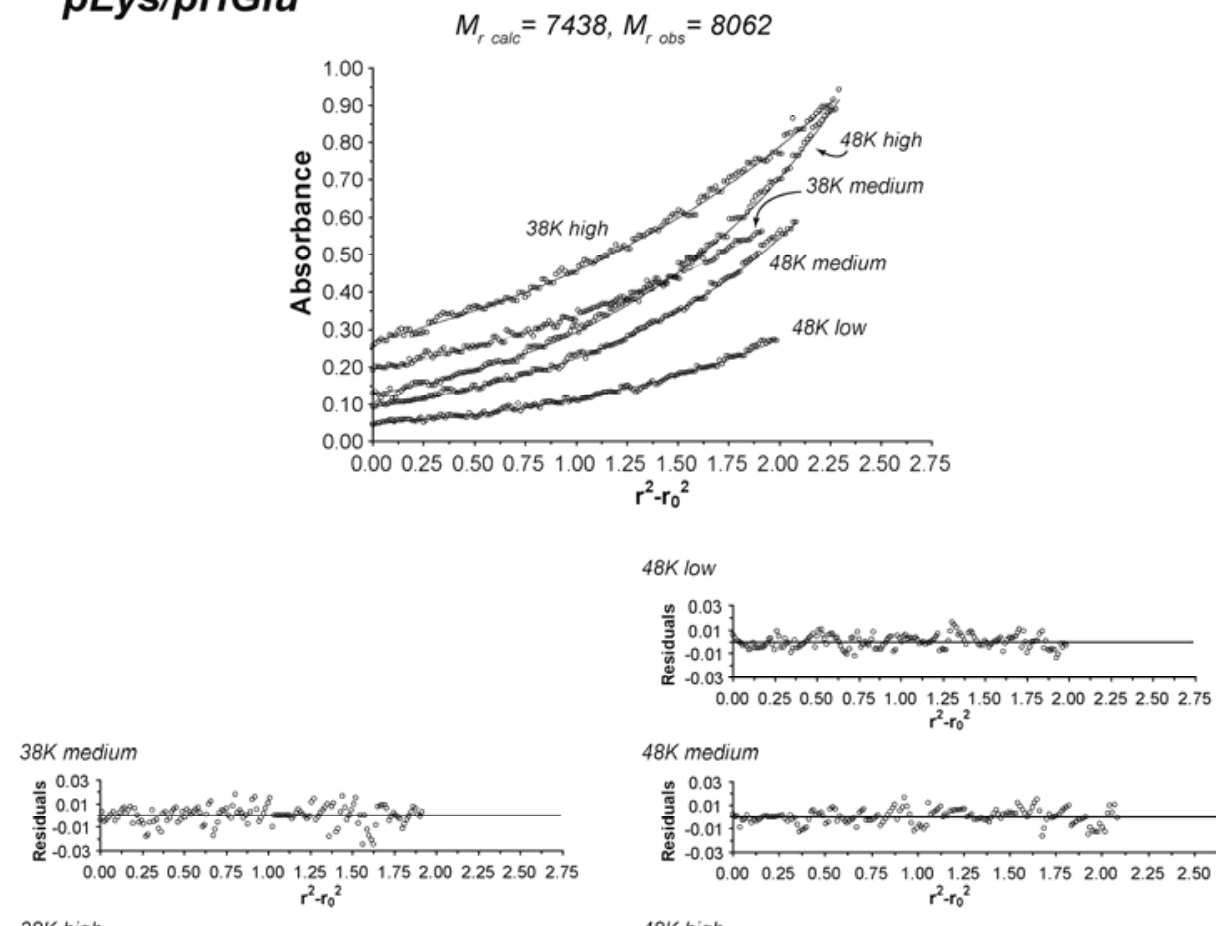

$38 \mathrm{~K}$ high

$48 \mathrm{~K}$ medium

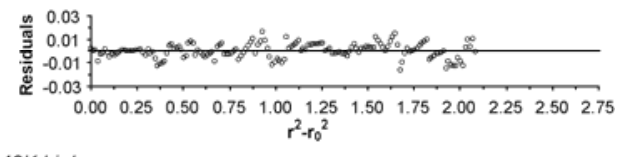

48K high
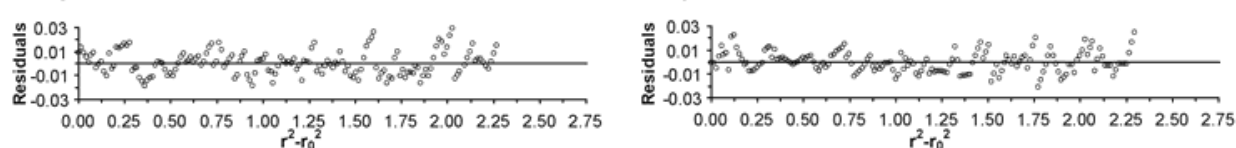

$r^{2}-r_{0}^{2}$ 
pDapt/pGlu

$M_{r \text { call }}=7216, M_{r \text { obs }}=6793$

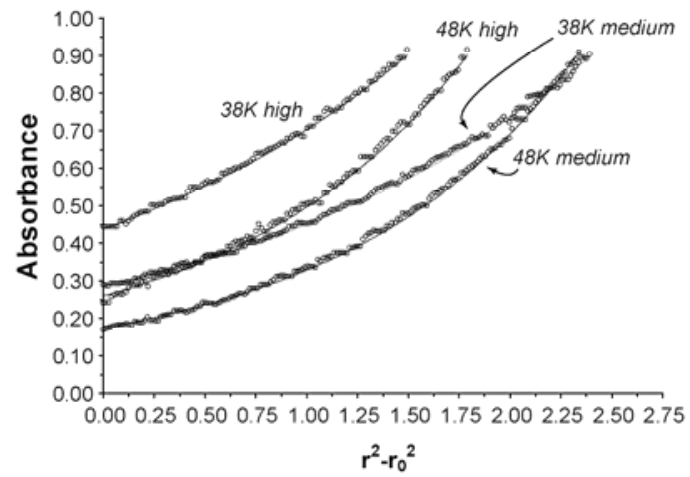

$38 \mathrm{~K}$ medium

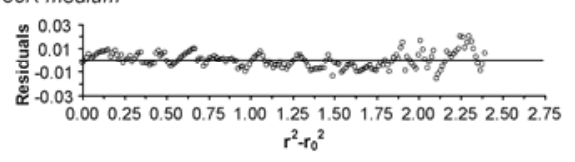

$38 K$ high

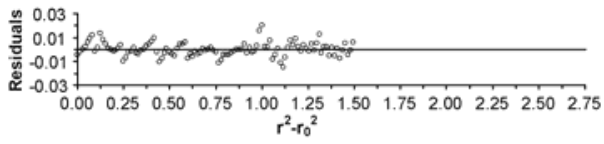

$48 \mathrm{~K}$ medium

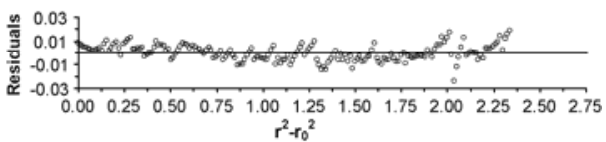

$48 \mathrm{~K}$ high

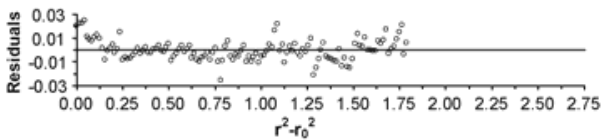

S-13 
pDab/pHHGlu

$$
M_{r \text { calc }}=7329, M_{r \text { obs }}=7747
$$

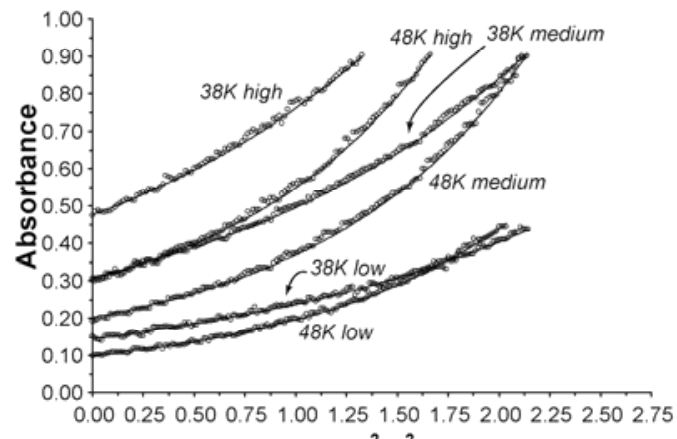

$$
r^{2}-r_{0}^{2}
$$

$38 \mathrm{~K}$ low

\section{8k low}

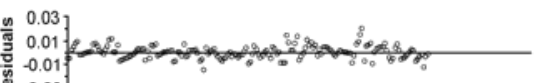

0.000 .250 .500 .751 .001 .251 .501 .752 .002 .252 .502 .75 $r^{2}-r_{0}^{2}$

$38 \mathrm{~K}$ medium

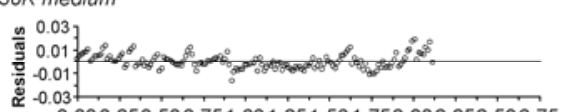

0.000 .250 .500 .751 .001 .251 .501 .752 .002 .252 .502 .75 $r^{2}-r_{0}^{2}$

$38 K$ high

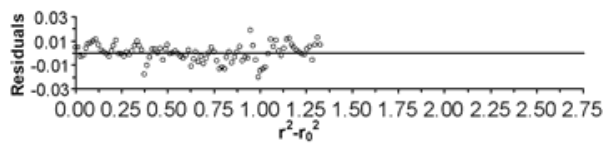

$48 \mathrm{~K} \mathrm{low}$

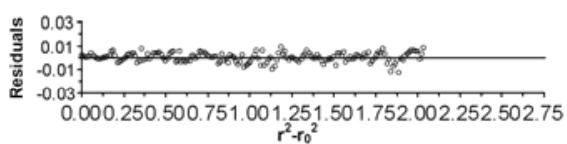

$48 \mathrm{~K}$ medium

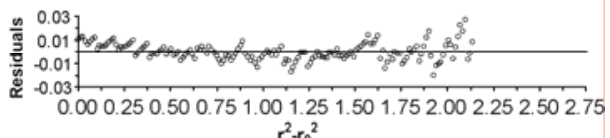

48K high

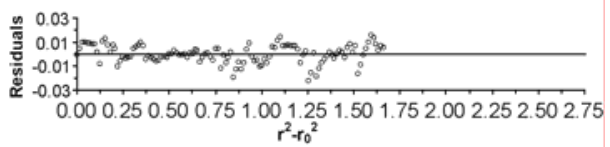




\section{pDapt/pHHGlu}

$$
M_{r \text { calc }}=7441, M_{r o b s}=7868
$$

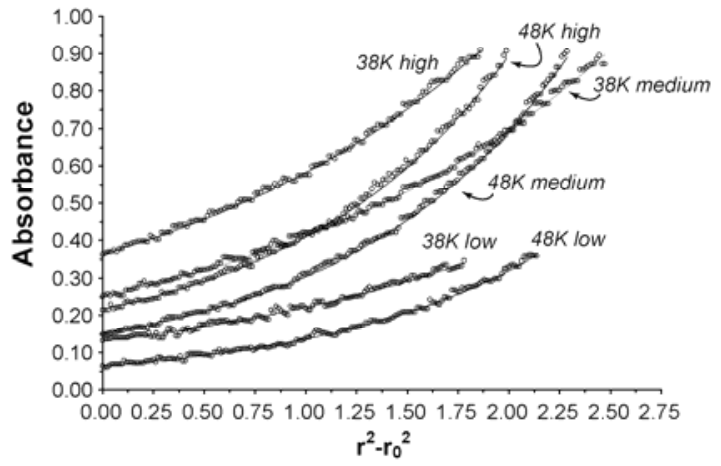

$38 \mathrm{~K} \mathrm{low}$

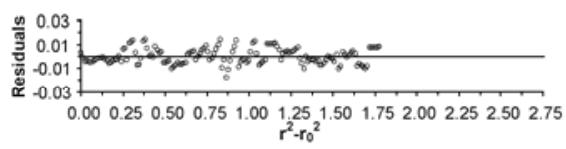

$38 \mathrm{~K}$ medium

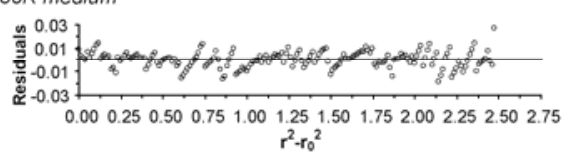

$38 \mathrm{~K}$ high

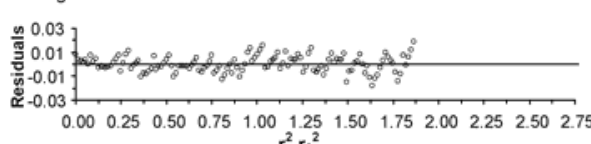

$48 \mathrm{~K}$ low

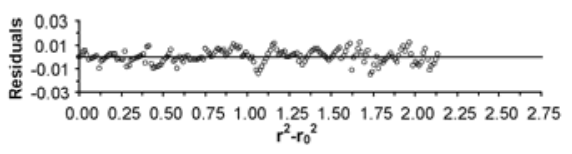

$48 K$ medium

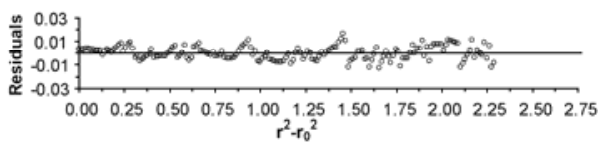

$48 \mathrm{~K}$ high

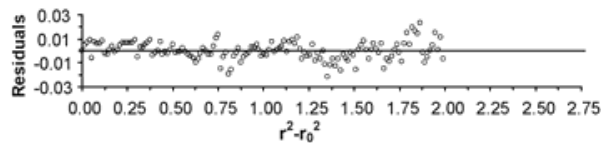




\section{pLys/pHHGlu}

$$
M_{r \text { calc }}=7553, M_{r \text { obs }}=7815
$$

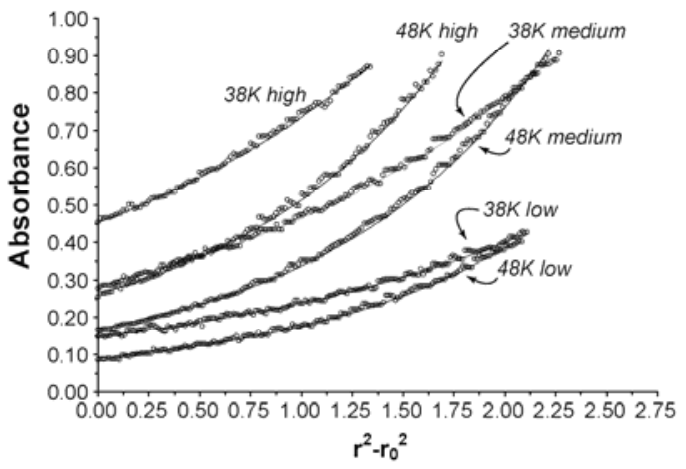

$38 \mathrm{~K}$ low

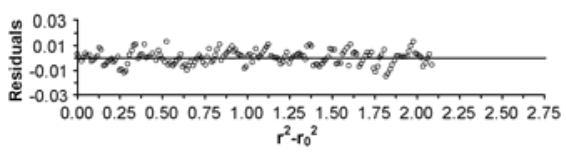

$38 \mathrm{~K}$ medium

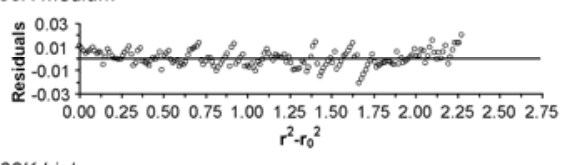

38K high

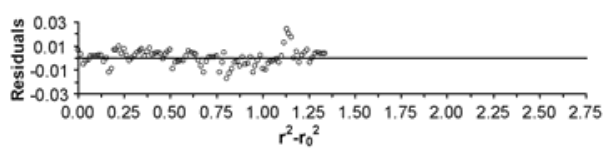

48 K low

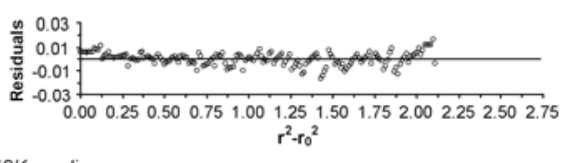

$48 \mathrm{~K}$ medium

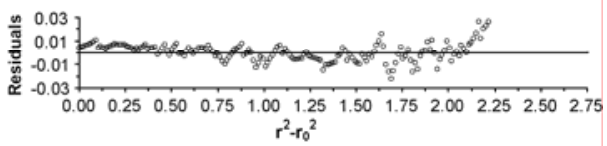

48K high

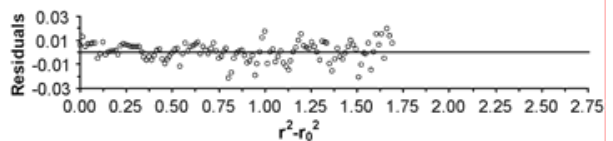

